\title{
Perspective
}

\section{Position Statement on Anti-Dementia Medication for Alzheimer's Disease by Swiss Stakeholders}

\author{
Giovanni B Frisoni ${ }^{1, * \mathbb{D}}$, Jean-Marie Annoni ${ }^{2}$, Stefanie Becker ${ }^{3}$, Tim Brockmann ${ }^{4}$, Markus Buerge ${ }^{5}$ (D) \\ Jean-François Démonet ${ }^{6}$, Dan Georgescu ${ }^{5,7}$, Anton Gietl ${ }^{8}$, Ulrich Hemmeter ${ }^{7}$, Stefan Klöppel ${ }^{9}$, \\ Thomas Leyhe ${ }^{10,+}$, Andreas U Monsch 11,12, Franco Rogantini ${ }^{13}$, Delphine Roulet Schwab ${ }^{14}$, Egemen Savaskan ${ }^{15}$, \\ Karl Schaller ${ }^{16}$, Armin von Gunten ${ }^{7}$ and Gabriel Gold ${ }^{1}$
}

check for

updates

Citation: Frisoni, G.B.; Annoni, J.-M.; Becker, S.; Brockmann, T.; Buerge, M.; Démonet, J.-F.; Georgescu, D.; Gietl,

A.; Hemmeter, U.; Klöppel, S.; et al.

Position Statement on Anti-Dementia Medication for Alzheimer's Disease by Swiss Stakeholders. Clin. Transl. Neurosci. 2021, 5, 14. https:// doi.org/10.3390/ctn5020014

Academic Editor: Claudio Bassetti

Received: 26 July 2021

Accepted: 9 August 2021

Published: 10 August 2021

Publisher's Note: MDPI stays neutral with regard to jurisdictional claims in published maps and institutional affiliations.

Copyright: (c) 2021 by the authors. Licensee MDPI, Basel, Switzerland. This article is an open access article distributed under the terms and conditions of the Creative Commons Attribution (CC BY) license (https:// creativecommons.org/licenses/by/ $4.0 /)$.
1 Centre de la Mémoire, HUG and University of Geneva, 1205 Geneva, Switzerland; gabriel.gold@hcuge.ch

2 Memory Clinic of the Canton Fribourg HFR FNPG and Fribourg University, 1700 Fribourg, Switzerland; jean-marie.annoni@unifr.ch

3 Alzheimer Switzerland, 3011 Bern, Switzerland; stefanie.becker@alz.ch

4 Association Suisse pour la Recherche sur l'Alzheimer (APRA), 1207 Genève, Switzerland; tbrockmann@recherchealzheimer.ch

5 Swiss Memory Clinics Association, 3000 Bern, Switzerland; markus.buerge@siloah.ch (M.B.); Dan.Georgescu@pdag.ch (D.G.)

6 Centre Leenaards de la Mémoire, CHUV and University of Lausanne, 1011 Lausanne, Switzerland; Jean-Francois.Demonet@chuv.ch

7 Swiss Society for Old Age Psychiatry and Psychotherapy (SGAP-SPPA), 6353 Weggis, Switzerland; Ulrich.Hemmeter@psgn.ch (U.H.); armin.von-gunten@chuv.ch (A.v.G.)

8 Center for Prevention and Dementia Therapy, Institute for Regenerative Medicine (IREM), University of Zurich, 8952 Schlieren, Switzerland; anton.gietl@irem.uzh.ch

9 Memory Clinic, University Hospital of Old Age Psychiatry (UPD), 3012 Bern, Switzerland; stefan.kloeppel@upd.ch

10 Geriatric Psychiatry, University Department of Geriatric Medicine and Psychiatric University Hospital, 4002 Basel, Switzerland

11 Swiss Association of Neuropsychologists (SVNP/ASNP), 3008 Bern, Switzerland; andreas.monsch@unibas.ch

12 Memory Clinic, University Department of Geriatric Medicine FELIX PLATTER, 4055 Basel, Switzerland

13 Synapsis Foundation-Alzheimer Research Switzerland ARS, 8005 Zürich, Switzerland; rogantini@alzheimer-synapsis.ch

14 Société Suisse de Gérontologie (SSG), 3097 Liebefeld, Switzerland; d.rouletschwab@ecolelasource.ch

15 Psychiatrische Universitätsklinik Zürich, Klinik für Alterspsychiatrie, 8032 Zürich, Switzerland; egemen.savaskan@puk.zh.ch

16 Swiss Federation of Clinical Neuro-Societies (SFCNS), 4001 Basel, Switzerland; karl.schaller@hcuge.ch

* Correspondence: giovanni.frisoni@hcuge.ch

† Professor Dr Thomas Leyhe sadly passed away in March 2021

Abstract: The present document represents the position of Swiss health-care associations, clinical and research centers, research-supporting foundations, and the association Alzheimer Switzerland regarding the care of persons with dementia and Alzheimer's disease. We claim that dementia is not part of normal aging but a disease developing more frequently in old age; early diagnosis and treatment of dementia is paramount; all patients with dementia have the right to receive state-ofthe-art treatments; more intense information, education, and counseling on dementia are necessary; media should provide balanced and fair reporting of scientific discoveries on Alzheimer's and dementia; all patients with dementia have the right to be treated; anti-dementia drugs should be used and accompanied by listening, compassion, and understanding.

Keywords: dementia; Alzheimer's; cholinesterase inhibitors

The number of dementia patients in Switzerland continues to rise sharply. There is also an increase of statements and guidelines from experts and professional societies, scientific evidence, and policy initiatives such as the 2014-2019 National Dementia Strategy, 
which addresses the effectiveness and quality of dementia treatment. Nevertheless, there are still parts of society questioning the efficacy and utility, in particular, of the medical treatment of dementia and even its very existence as a medical condition.

These voices have also led to skepticism at the level of some health authorities regarding the effectiveness of drugs in the treatment of dementia. For example, in France, the costs of officially approved anti-dementia drugs (medicines that can relieve various symptoms of Alzheimer's disease) are no longer reimbursed by health insurance. But given the many failures in clinical drug research, the importance of available pharmacological treatments as well as psychosocial approaches is again underlined.

This view and the associated consequences contradict all facts and the current knowledge about diseases associated with dementia.

The present document represents the position of the relevant health-care associations, clinical and research centers, research-supporting foundations, and the association Alzheimer Switzerland.

Dementia is not part of normal aging but a disease developing more frequently in old age. The term dementia includes diseases of various causes, which are distinguished by decreased cognitive function, limitations of everyday activities, and behavioral disturbances (depression, apathy, irritability, insomnia, etc.). Causes of dementing diseases may be predominantly of a neurodegenerative (in particular Alzheimer's disease, dementia with Lewy bodies, frontotemporal dementia) or vascular nature (diseases of the small or large brain arteries). These conditions are listed in the International Classification of Diseases (ICD-10), which also provides the basis for reimbursement of the basic health-care insurance (KVG/LAMal). Depending on the etiology of dementia, different treatment options are available, including four officially approved anti-dementia drugs. In order to achieve maximum benefit, early and accurate diagnosis is paramount.

All patients with dementia have the right to receive state-of-the-art treatments. Even in Western countries, between $40 \%$ and $70 \%$ of patients with dementia still go undetected. Others do not get a proper clinical examination by experienced physicians and further imaging and laboratory investigations are often not performed even if a neurodegenerative disease is suspected. This prevents appropriate interventions of preventable or reversible forms of dementia (i.e., vascular dementia, neuroinflammation, recurrent seizures, chronic sleep-wake disorders, etc.).

In some nonreversible forms of dementia, pharmacologic and non-pharmacologic options are available, as described in numberless medical textbooks. Therapy with currently available anti-dementia drugs together with non-pharmacologic options can improve cognitive deficits and behavioral disturbances, facilitate emotional stabilization, and help patients to remain in their social environment. Although not curative, currently available drugs have demonstrated efficacy to improve or stabilize cognitive symptoms for as long as 18 months and deceleration of cognitive worsening for as long as 5 years. In recent years, the knowledge of the causes of Alzheimer's disease has expanded enormously, and new therapeutic approaches are being developed in preclinical and clinical research. For this reason, it is vital to continue investing in research to develop new pharmacological and non-pharmacological treatment options.

Treatment should not be confined to core dementia symptoms but should extend to comorbidities within and outside the central nervous system, such as seizures, depression, sleep-wake disorders, constipation, and low caloric intake, all of which have a significant impact on patients' quality of life. Importantly, these considerations will hold even in a treatment space where diseased modifiers such as anti-amyloid monoclonal antibodies are available.

Early diagnosis and treatment of dementia is crucial. The number of patients with dementia will continue to increase in the next years, not least due to demographic changes (population aging). Early detection and treatment enable longer term autonomy and stabilization at home and delay long-term nursing home placement. It thus reduces the burden on relatives and the societal costs of care. Early detection is also the prerequisite for 
patients to decide on their own treatment and care in the present and in their future (i.e., advanced care planning). For these reasons, patients with diagnosed dementia must be treated quickly and in accordance with the latest state-of-the art Swiss and international guidelines. Treatment should include anti-dementia drugs in patients with Alzheimer'stype dementia whenever possible.

More intense information, education, and counseling on dementia are necessary. Currently, a large part of dementia cases is not diagnosed or diagnosed too late, and only a fraction is treated adequately. To address this issue, promoting awareness of the general population, counseling of the affected persons and their relatives, education to health-care professionals of pharmacological and psychosocial interventions, and networking among professionals must be placed at the forefront of the political agenda. Only in this way can an optimization of the efficiency and a reduction of the costs of care be achieved in the context of the currently available treatments. Denying treatment to patients in the early disease stage ultimately leads to higher health costs in the more severe later stages of the disease.

Prescribing drugs should be accompanied by listening, compassion, and understanding. Alzheimer's and related diseases hit the very core of humanity of a person and what they represent for their dear ones, friends, and colleagues. Patients with Alzheimer's and other dementing diseases have the right to be cared for by empathic, attentive, patient, and listening health-care professionals. Far from being alternative, the biomedical and the psychosocial approaches enrich and complement each other, and the result is much more than the sum of the two.

Media should provide balanced and fair reporting of scientific discoveries on Alzheimer's and dementia. We understand and share the disappointment of the civil society at the inconsiderate use of premature scientific results on new drugs for Alzheimer's, often at very early development stages, or fake curative interventions. We also share the disappointment at the pessimism arising from failures of drugs aimed at slowing the progression or preventing the development of the disease. Even the most successful drugs need between 5 and 10 years from evidence of efficacy in animal models to availability to patients and, even if successful, will only be adequate for a certain group of patients. We advocate a cautious approach of media and scientists alike when delivering information on scientific discoveries in the Alzheimer's drug domain.

All principles expressed above were also part of the National Dementia Strategy 2014-2019 of the Swiss Federal Government, and the Cantons, and are strongly endorsed by the signatories of this article.

Author Contributions: G.B.F. drafted the first version of the manuscript. All other authors revised the draft and contributed important intellectual content. All authors have read and agreed to the published version of the manuscript.

Funding: The authors received no financial support for the research, authorship, and/or publication of this article.

Institutional Review Board Statement: Not applicable.

Informed Consent Statement: Not applicable.

Data Availability Statement: Not applicable.

Conflicts of Interest: The authors declared the following potential conflicts of interest with respect to the research, authorship, and/or publication of this article: The lead author as well as most coauthors who are also memory clinic directors have been and currently are involved in clinical trials of anti-dementia drugs. None currently has any competing interest with the marketing or continuing development of licensed anti-dementia drugs - the core topic of this piece. 